\title{
Einleitung zur Neuausgabe
}

Kaffee ist unser beliebtestes Getränk, beliebter noch als Mineralwasser. Jeder Bundesbürger verbraucht im Monat durchschnittlich rund ein Pfund Röstkaffee, das entspricht etwa der Gesamternte eines Kaffeebaumes. Zwölf Kaffeebäume werden für jeden von uns pro Jahr von Hand geerntet. Die Kaffeebohnen werden aus den Kirschen gestreift, gereinigt und getrocknet. Dann muss der Kaffee noch eine lange Reise zurücklegen - denn der Baum wächst nur in einem schmalen Gürtel rund um den Äquator. Wenn die Bohnen hier angekommen sind, werden sie noch geröstet, gemahlen und verpackt - so kaufen wir sie dann an der Ladentheke. Gar nicht wenig Aufwand für ein alltägliches Getränk.

Deutschland importiert rund 15 Prozent der weltweiten Kaffeeernte und ist damit nach den USA der zweitwichtigste Importeur. Und nicht nur Deutsche und Amerikaner lieben den Kaffee - weltweit wird er geschätzt! Kein Wunder, dass Kaffee zu den wichtigsten Handelswaren der Welt zählt. Für lange Zeit war er sogar der zweitwichtigste Rohstoff auf dem Weltmarkt - übertroffen nur vom Rohöl. Etwa 25 Millionen Bauern leben mit ihren Familien von der Produktion dieses Stoffes. Nur sehr wenige Agrarprodukte haben eine vergleichbare wirtschaftliche Bedeutung.

Zugleich haben nur sehr wenige Stoffe eine ähnliche kulturelle Strahlkraft. Seit der Kaffee in Europa angekommen ist, seit etwa fünfhundert Jahren, hat er die Welt um ihn herum verwandelt. Er löste die Alkoholika Bier und Wein als Hauptgetränk ab und wurde Begleiter einer nüchterneren Epoche. Was die Aufklärung auf geistigem Weg zu bewerkstelligen suchte, unterstützte er physiologisch. Zugleich ist der Kaffee ein hochgradig politischer Stoff. Massive Auseinandersetzungen begleiten seinen Weg. Er gab das Stichwort zu Diskussionen über Sklaverei, Ausbeutung und Gerechtigkeit und über das Nord-Süd-Verhältnis. Über den Kaffee sind wir verbunden mit Menschen in weit entfernt liegenden Teilen der Welt: Jede Kaffeebohne hat einen weiten Weg durch den Raum hinter sich - wie der Kaffee als solcher einen langen Weg durch die Zeiten und Gesellschaften zurückgelegt hat.

Kaffee ist vielfach Gegenstand historischer Untersuchungen geworden. Zusammenfassende Synthesen der Geschichte des Kaffees sind dennoch 
selten. Die meisten historischen Darstellungen beschränken sich auf einen bestimmten Aspekt, sei es die Konsumgeschichte oder auch eine geographische Produktionsregion. Erst die Zusammenschau aber ermöglicht ein umfassendes Verständnis der »Welt des Kaffees«. Und eben diese Zusammenschau ist das Ziel von Heinrich Eduard Jacobs Werk über den Kaffee, das 1934 erstmals erschienen ist.

Gäbe es einen Kanon der klassischen Sachbücher, dieses Buch gehörte dazu - weil es auf eine neuartige Weise eine Sache, einen Stoff in den Mittelpunkt einer romanhaften Geschichte gestellt hat. Dabei befasst Jacob sich nicht nur mit der Konsumgeschichte des Kaffees, sondern auch mit seiner Produktion und mit dem Handel, der beide miteinander vermittelt. Zugleich geht es dem Autor ums Erzählen. Schon der Ausgangspunkt, die mit viel Liebe instrumentierte Legende von der Entdeckung des Kaffees durch ein paar Hirten aus dem Jemen zeigt, gibt die Richtung vor: Nicht nur eine berichtartige Darstellung der Fakten will Jacob liefern, sondern eine mit klassischer Technik erzählte Geschichte.

Jacobs Buch erschien damals in den dreißiger Jahren unter dem ungewöhnlichen Titel: Sage und Siegeszug des Kaffees. Die Biographie eines weltwirtschaftlichen Stoffes. Von der zeitgenössischen Kritik wurde es durchweg freundlich, oft euphorisch begrüßt, obwohl in der Berliner Börsenzeitung und im Börsenblatt zum Boykott des jüdischen Autors aufgerufen worden war. Namhafte Kritiker ließen sich davon nicht einschüchtern. So lobte Hermann Hesse im schweizerischen Berner Bund, dass Jacob eben nicht "zu jenen Halbliteraten " gehöre, »denen ansonsten das Verfassen ähnlicher Monographien überlassen bleibt«, sondern »ein durchaus ernst zu nehmender Autor ..., ein Dichter" sei.

Freilich halfen solche Besprechungen dem Buch im nationalsozialistischen Deutschen Reich nichts. 1935 wurde Jacobs gesamtes Werk verboten, 1938, nach dem "Anschluss Österreichs « wurde Jacob in Wien verhaftet und ins KZ Dachau verschleppt. Dank des unermüdlichen Einsatzes seiner Verlobten und späteren Frau Dora Angel-Soyka kam Jacob nach einem Jahr schwer erkrankt und mit inneren Verletzungen - wieder frei; unmittelbar darauf emigrierte er in die USA. Nach dem Krieg kehrte er zurück, konnte jedoch im Nachkriegsdeutschland nicht mehr richtig Fuß fassen und lebte ein unstetes Wanderleben von Hotel zu Pension. 1967 starb er in Salzburg.

Weil die Kapitel der Kaffeegeschichte in vielen verschiedenen Ländern spielen, musste Jacob bei seiner Recherche auf ganz unterschiedliche nationale Literaturen zurückgreifen. Und weil zudem der Kaffee die unterschiedlichsten Wissensgebiete durchquert, von der Chemie über Geographie, Volkswirtschaftslehre bis hin zur Soziologie, galt es auch in verschiedenen Disziplinen zu forschen. Fünf Jahre, schreibt Jacob, habe er für das Werk recherchiert - wenn auch sicher nicht ausschließlich, da er hauptberuflich

10 Einleitung zur Neuausgabe 
als Journalist für das Berliner Tageblatt arbeitete. 1932 unternahm er im Zuge seiner Recherchen an dem Buch eine Reise nach Brasilien, damals wie heute Hauptexporteur des Kaffees: mit einem Zeppelin, von Friedrichshafen nach Recife in Nordost-Brasilien.

Das Kaffeebuch von Heinrich Eduard Jacob schlägt die Brücke zu älteren Werken, die von aktuellen Kaffeehistorien fast immer übergangen werden. Für die europäische Geschichte des Kaffees ist es auch heute noch ein unentbehrlicher Begleiter. Es sind vor allem die jungen Jahre des Kaffees in Europa, die Jacob in seinem Werk ausführlich darstellt. Sie sind auch historisch entscheidend, da sich in diesen Jahren die Bahnungen herausbilden, in denen sich die Aneignung dieses Genussmittels vollzieht, seine Vermittlung mit der eigenen Kultur. Zu dieser Vermittlung hat auch Jacobs Buch selbst beigetragen, wie kein früheres und kein späteres Werk. In fast allen neueren Büchern über den Kaffee stößt man auf seinen Einfluss. Jacobs Buch ist selbst Teil der Geschichte des Kaffees geworden.

Mit ein Grund, dieses über Jahrzehnte vergriffene Grundlagenwerk der Kaffeehistorie im Rahmen der Reihe Stoffgeschichten der heutigen Leserschaft wieder zugänglich zu machen, ist die - damals wie heute - ungewöhnliche literarische Herangehensweise Jacobs. Sein Buch hat jener literarischen Tradition, der sich die Stoffgeschichten verpflichtet fühlen, bedeutende Impulse gegeben: Es ist eines der ersten Bücher, die einen Stoff in den Mittelpunkt einer Geschichte stellen und literarische Formen entwickelt haben, um über einen so ungewöhnlichen »Helden« wie den Kaffee zu erzählen. Jacob war sich dieser Leistung bewusst, und sie ist auch in der Literatur oft gewürdigt worden: Nicht umsonst bezeichnet die Neue Deutsche Enzyklopädie Jacob als den »Vater des Neuen Sachbuchs«.

Aber ist sein Werk inhaltlich noch aktuell? Oder ist es im Laufe der Jahre nicht schal geworden - »kalter Kaffee«? Haben neuere Erkenntnisse nicht alles, was Jacob in den dreißiger Jahren mühevoll recherchiert hat, längst überholt? Solche Fragen sind nahe liegend.

Jacobs Buch weist streckenweise Spuren des Alters auf, die manche Seiten für den heutigen Leser dissonant oder auch grell klingen lassen, etwa, wenn der unverbesserliche Bürger Jacob vom »armen Volk« spricht, »dem schließlich alles gleichgültig ist«. Bisweilen irritiert es, wenn Jacob sich als Völkerversteher in Szene setzt und über »den« Engländer oder »den« Niederländer oder die »kindlichen Einwohner « Indonesiens schreibt - und mehr noch, wenn er sich als Frauenversteher zu erkennen gibt und vermutet, dass die "gehirnliche Wirkung« des Kaffees dem »Harmoniebedürfnis gerade der besten Frauen« möglicherweise abträglich sei. Formuliert Jacob etwa selbst in jenem selbstgefälligen chauvinistischen Tonfall, der in den dreißiger Jahren mit schrecklichen Folgen Raum griff? 
Jacob war ein Kind seiner Zeit. Zugleich war er aber ein hellsichtiger Kritiker seiner Zeit. Sein Werk über den Kaffee ist kein nationalistisches Epos, sondern ein leidenschaftliches Plädoyer für Weltoffenheit. Das zeigt besonders das erste "Buch« Der Wein des Islam, in dem er über die arabische und türkische Kultur schreibt. Die Textpassagen über die Belagerung von Wien durch die Türken und die anschließende Schlacht im Jahre 1683 sind ohne kulturelle Arroganz formuliert. Um seine Aussage einordnen zu können, muss man sich daran erinnern, wie die Belagerung Wiens zu jener Zeit, in der dieses Kapitel entstand, sonst dargestellt wurden. 1933 wurde in Wien der 250. Jahrestag der Befreiung gefeiert - und zehntausende austrofaschistische und nationalsozialistische Wiener machten die Feiern zum Anlass für Hetze gegen die Türken, Juden und andere Fremde. Der Kosmopolit Jacob hingegen liefert eine von Ressentiments freie Darstellung der Herkunft jenes Getränks, das für ihn mit der modernen, aufgeklärten Zivilisation auf das Innigste verknüpft ist. In seiner Verbindung von erzählerischer Technik und verlässlicher Information bleibt Jacobs Buch eine hervorragende Spezialiät auf dem Markt der Kaffeehistorien. Entstanden ist eine, man möchte sagen, anmutige Synthese, die den Leser informiert und unterhält.

Seine Sprache wirkt oft pathetisch, sie lässt kaum eine rhetorische Figur aus und ist reich an Ornamenten. Manche Sätze trippeln daher wie Primadonnen und scheinen auf Applaus zu warten. Für einen Leser, der nüchterne Prosa bevorzugt, bisweilen eine störende Virtuosität. Zu Recht weist die Literaturwissenschaftlerin Anja Clarenbach, die eine umfassende Monographie über Jacob vorgelegt hat, auf die manchmal allzu hochtourige Sprache seines Werkes hin. Sie erinnert aber an eine Anmerkung Jacobs, dass ein Buch über eine vermeintlich tote Materie hymnisch geschrieben sein müsse, weil nur so der "Sachwelt«, in der wir leben, »ihre Würde zurückzugeben«sei.

Die fünf Bücher, in die das Werk kapitelweise aufgeteilt ist, sind unaufdringlich chronologisch: Im ersten Teil (Der Wein des Islam) erzählt Jacob die Ausbreitung des Kaffees in der islamischen Welt. Die Ursprünge verlegt er dabei etwa ins 9. Jahrhundert nach Christi und geographisch ins Land Yemen - was sich mit heutigen archäologischen Befunden in Übereinstimmung bringen läßt, auch wenn wir kaum Zeugnisse aus der frühen Geschichte des Kaffees besitzen - erst die Zeit ab 1500 ist gut dokumentiert. Yemen wurde von den Türken erobert, die dem Kaffeegenuss aufgeschlossen waren und mit dem Stoff Handel trieben. Jacob beschließt das erste Buch mit der Geschichte um Georg Franz Kolschitzky, der, wie Jacob nacherzählt, das erste Wiener Kaffeehaus gegründet haben soll. (Hier sitzt Jacob allerdings einer Legende auf: Das erste Wiener Kaffeehaus wurde 1685 von dem Armenier Johannes Diodato eröffnet.) Auch wenn Wiens Kaffeehäuser berühmt wurden - es waren keineswegs die ersten Kaffeehäuser Europas,

12 Einleitung zur Neuausgabe 
sondern Spätlinge. Es ist allerdings möglich, dass in Wien aus dem Getränk durch Zusatz von Honig und Sahne etwas wurde, das den europäischen Gaumen besser mundete als das Original, das schwarz, ungefiltert und ungesüßt genossen wurde.

Unter dem Titel Die Gesundheit der Nationen verfolgt Jacob des Weg des Kaffees in Nord- und Südeuropa und beschreibt die Abstoßungsreaktionen und Angriffe, denen das neue, wachmachende Getränk in einer Umgebung, die vor allem Bier und Wein trank, zunächst ausgesetzt war.

Anschließend, unter der Überschrift Pflanzer, Krämer, Könige beschreibt er im dritten Buch die ersten Kaffeeplantagen, die im 17. Jahrhundert in niederländischen Kolonien gesetzt wurden: Hier liegen die Anfänge der modernen Kaffeeökonomie. Zugleich verfolgt Jacob die Geschichte des Kaffees durch das 18. Jahrhundert, wobei er sich vor allem auf den Konsum konzentriert.

Schließlich betrachtet er den Kaffee und das Neunzehnte Jahrhundert, wobei sich sein Blick verengt: Er betrachtet nur die Kaffeekultur in Europa, besonders in Preußen und Österreich, Nordamerika bleibt bis auf wenige Anmerkungen ausgeklammert. (Das ist insofern berechtigt gewesen, als die Europäer damals die hauptsächlichen Konsumenten des Kaffees waren. Erst um die Wende zum 20. Jahrhundert entwickelt sich Nordamerika zum Hauptimporteur.) Dann wendet er im letzten Abschnitt des vierten Buches den Blick auf Produktion und Börsenhandel: eine brillante Darstellung der internationalen Kaffeeökonomie.

Da diese Ökonomie zu Jacobs Zeiten vom Hauptproduzenten Brasilien dominiert wird, widmet sich das Schlusskapitel Die Diktatur Brasiliens der Geschichte des Kaffees in Brasilien: bis hin zur großen Ernteverbrennung in den dreißiger Jahren, ein auch aus heutiger Sicht außergewöhnliches Ereignis, das Jacob als Augenzeuge beschreibt. Mit einem >Abgesang « endet dieses höchst ungewöhnliche, nicht nur inhaltlich, sondern auch formal ambitionierte Sachbuch. Später ergänzte er sein Buch anlässlich dessen zweiter Auflage um eine Nachschrift, welche die Kaffeegeschichte bis Anfang der fünfziger Jahre weitererzählt.

Buch von Heinrich Eduard Jacob ist in zwei deutschen Auflagen und einer Taschenbuchausgabe erschienen, deren Text sich geringfügig unterscheidet. Unsere Ausgabe folgt dem Text der späteren Auflage von 1952. Auch ein Großteil der Illustrationen haben wir übernommen. Der Titel wurde leicht verändert, wobei wir uns am Titel der amerikanischen Ausgabe orientiert haben, die vor wenigen Jahren erneut erschienen ist (Coffee: The Epic of a Commodity). Ergänzt wird das Buch im Anhang um zwei Texte, in denen zum einen die weitere Kaffeegeschichte bis hin zur Gegenwart präsentiert wird, zum anderen das Werk Jacobs in den Kontext seiner Entstehung ein- 
geordnet und hinsichtlich seiner Erzählstruktur analysiert wird. Ein wichtiges Supplement sind die Karten, die von den Augsburger Geographen Simon Meißner und Thomas Staudinger angefertigt wurden und in einem gesonderten Bildteil dokumentiert sind. Sie zeichnen die Wege des Kaffees durch Raum und Zeit graphisch nach und liefern eine sinnliche Ergänzung zu jener Geschichte, die Jacob arabeskenreich erzählt.

Danken möchten wir Gabriele Bihler von der Universitätsbibliothek der Universität Augsburg, die uns bei der Beschaffung der umfangreichen Literatur zu Stoffen und speziell zum Kaffee unterstützt hat; Hans Jörgen Gerlach, dem Nachlaßverwalter von Heinrich Eduard Jacob, der umfangreiches Archivmaterial von und über Jacob zur Verfügung stellte und die Neuherausgabe dieses Buches vielfältig unterstütze; Kerstin Gluth, die mit kritischen Rückfragen geholfen hat, dass viele Formulierungen klarer wurden, und ganz besonders Manuel Schneider, der entscheidend dazu beigetragen hat, dass die Neuauflage möglich wurde, und sie als Lektor betreut hat. Nicht zuletzt verdanken wir ihm auch wichtige Anregungen für die Gestaltung der ergänzenden Texte.

Augsburg, im September 2006

Armin Reller und Jens Soentgen

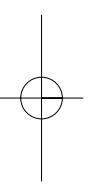

Titelbild der Erstausgabe "Sage und Siegeszug des Kaffees. Die Biographie eines weltwirtschaftlichen Stoffes« von 1934.

14 Einleitung zur Neuausgabe 